\title{
Diagnostic and prognostic roles of miRNA and dielectric parameters in HCV Egyptian patients
}

\author{
Doha Mohammad Beltagy*, Yasser Ibrahim Khedr, Abdel-Hamid A. Sakr \\ Faculty of Science, Damanhour University, Damanhour, Egypt.
}

\section{ARTICLE INFO \\ Received on: 27/04/2020 \\ Accepted on: 21/06/2020 \\ Available online: 05/09/2020}

\section{Key words:}

Hepatitis, miRNAs, Sovaldi, dielectric parameters, Ribavirin.

\begin{abstract}
Hepatic $\mathrm{C}$ virus (HCV) infection is a special case in Egypt due to its historical aspects, epidemiology, risk factors, and genotype. Therefore, an urgent need arose to assess the HCV progression and efficacy of the used antiviral drugs. This study aimed to evaluate the role of different miRNAs types and blood dielectric measurements in the early diagnosis and prognosis of HCV in Egyptian patients. The study was carried out on a total of 80 blood samples. Twenty of these blood samples were withdrawn from healthy volunteers and were served as the control group (G1). Sixty HCV patient samples were divided according to the received treatment into four groups (15 for each). The second group (G2) included HCV patient samples, who did not receive any treatment. The third (G3), fourth (G4), and fifth (G5) groups included the samples of HCV patients who were treated with Sovaldi $(400 \mathrm{mg})$ for 1, 2, and 3 months, respectively. G3, G4, and G5 were simultaneously administered Ribavirin $(200 \mathrm{mg})$ and Daclatasvir $(60 \mathrm{mg})$ daily for 3 months. The obtained results demonstrated the upregulation of miR-21, miR-155, and miR-205 and downregulation of miR-122 and miR-133a in all HCV patients. HCV patients who did not receive any treatment showed a lower conductivity due to the action of the virus; either the free charges on the surface of the red blood cells (RBCs) or membrane deformation or alterations were reduced. Noticeable improvement reached to nearly normal values in different miRNA expressions and RBC dielectric relaxations was achieved by Sovaldi treatment.
\end{abstract}

\section{INTRODUCTION}

Hepatic $\mathrm{C}$ virus (HCV) infection has been considered for many decades as the most challenging health issue in Egypt. It represented the most common liver disease load. The HCV prevalence in Egypt is the highest in the world for many years, where it is a special case in all aspects such as history, epidemiology, risk factors, genotype, and treatment protocols (El-Ghitany, 2019). About 10.4 million Egyptian patients are infected with genotype four of $\mathrm{HCV}$, representing about $13 \%$ of total infections in the world (Ahmed et al., 2018).

The Micro ribonucleic acids (miRNAs) are highly maintained in almost all organisms. They are defined as a class of noncoding RNAs, about 18-25 nucleotides long, and play important roles in the regulation of gene expression. These

\section{"Corresponding Author}

Doha Mohammad Beltagy, Faculty of Science, Damanhour University, Damanhur, Egypt.E-mail: dohabel4@yahoo.com
miRNAs represent only $1 \%$ of the human genome. It was firstly discovered in 1993 (Bartel, 2009).

Recently, circulating miRNAs in biofluids as serum are used as biomarkers for different diseases due to their stability and noninvasive nature. Since circulating miRNAs show consistent levels between healthy people but significantly altered patterns in pathological conditions, the substantial efforts were made to study the changes in the circulating miRNA pattern in $\mathrm{HCV}$ infection and associated liver disorders (Lee et al., 2017).

Human blood electrical properties play important roles in determining the blood flow pathways inside the body and in the diagnosis and treatment of various physiological conditions. Therefore, on a more important level, understanding these electrical properties will result in more explaining of the basic biological processes. In turn, these studies are of great importance in electrophysiology and biophysics (Jaferzadeh and Moon, 2015). Electrical properties were previously used in various diseases or medical issues such as biofluid shift, cardiac output, and muscular dystrophy by using many impedance diagnostic techniques, such 
as rheoencephalography, cardiograph, and plethysmography (Livshits et al. 2006; Barshtein et al., 2011).

Moreover, electrical properties depend on all blood components, not only cells but also the various biocomplexes as proteins, salts, saccharides, hormones, vitamins, and antibodies. All these biocomplexes are lighter than blood cells. In general, the more the time, the greater the conductivity with slower changes in the frequency ranging over $100 \mathrm{~Hz}$. Taking these causes into consideration, some precautions should be taken in the data analysis of electrical measurements carried out on blood (BenIshai et al., 2013).

There were few recent studies focused only with the role of some miRNA types in hepatic viruses' life cycle and their regulated participation in $\mathrm{HCV}$-related liver disease development (Shrivastava et al., 2015). These studies suggested the ability to use some miRNA types as noninvasive biomarkers in liver damage, especially caused by HCV (El-Ahwany et al., 2019). An urgent need arose to early diagnose HCV and assess the efficacy of the used new antiviral drugs as Sovaldi in HCV treatment. This study aimed to assess the role of different types of miRNAs and blood dielectric properties in the early diagnosis and prognosis of $\mathrm{HCV}$ in Egyptian patients.

\section{SUBJECTS AND METHODS}

The present study was carried out on a total of 80 blood samples. Blood samples of 20 healthy volunteers were served as the negative control group (G1). Sixty HCV patient samples were divided according to the treatment received into four groups (15 for each). The second group (G2) included HCV patient samples who did not receive any treatment (zero-time dose). The third (G3), fourth (G4), and fifth (G5) groups were the samples of HCV patients, who were treated with Sovaldi (Sofosbuvir, $400 \mathrm{mg}$ ) for 1,2 , and 3 months (the end of treatment course), respectively. Patients in G3, G4, and G5 were administered Ribavirin (200 $\mathrm{mg}$ ) and Daclatasvir $(60 \mathrm{mg})$ daily for 12 weeks in addition to Sovaldi.

The HCV patient samples were admitted from the National Institute of Oncology, Ministry of Health, Damanhour City, Egypt, from October 2018 to April 2019. The clinical history of each study participant was recorded with extraordinary concern regarding any related clinical issues. All participants were subjected to full clinical examination and did not show clinical symptoms or signs of any other problems except hepatitis. The study protocol was revised and approved by the Ministry of Medicine, Egypt. Written informed consent was obtained from each patient. Privacy of the information was kept by making code numbers (accessible to only researchers) for each patient. All information were analyzed anonymously.

\section{Inclusion criteria}

All included subjects in the study aged from 18 to 55 years, of both sexes, and they were suffering from only HCV. Diagnosis mainly depended on HCV screening using HCV PCR. Liver function tests (such as ALT, AST, albumin, ALP, prothrombin, and total bilirubin) for all patients were within normal ranges. Among all the 60 patients who completed the treatment course (3 months), 60 patients (100\%) achieved negative HCV PCR.

\section{Exclusion criteria}

Restricted rules were established to exclude some individuals from the study: (a) aged below 18 years; (b) suffering from any preexisting chronic diseases other than HCV such as diabetes, hypertension, neoplasms, and renal or cardiovascular disorders; (c) being with abnormal liver function tests such as Alanine Aminotransferase (ALT), Aspartate aminotransferase (AST), albumin, Alkaline phosphatase (ALP), prothrombin, and total bilirubin; and (d) previously treated with any other types of medications. Full historical and personal data were registered in a separate sheet for each participant.

\section{Sample collection}

Blood samples were collected from patients at zero time (before administration of any medication). Repeated blood samples were also obtained after 1 month, 2 months, and 3 months of treatment from all subjects. Blood samples were collected from the antecubital vein in consideration with the sterilized conditions on two different tubes: (a) Ethylenediamine tetraacetic acid (EDTA-containing tubes for dielectric measurement and (b) empty tube, and then, they were immediately centrifuged at 5,000 rpm to obtain serum. The serum of each sample was then frozen at $-80^{\circ} \mathrm{C}$ till use in further analysis. Blood samples from 20 healthy volunteers were also obtained by the same way for once.

\section{MicroRNA isolation and detection using quantitative real- time PCR (qRT-PCR)}

MicroRNA was purified from serum samples of all experiment groups using the Direct-zol ${ }^{\mathrm{TM}}$ RNAMiniPrep compatible with TRI Reagent ${ }^{\circledR}$ (Catalog number R2050, ZYMO Research CORP, Irvine, CA). The samples were lysed and homogenized in lysis buffer of TRI Reagent, which contains phenol and guanidine thiocyanate solution to give the fast and best inhibition of RNase activity. The complete removal of DNA was performed by using DNase I digestion. miRNA purification and concentration were detected by nanodrop (A260/A280 > 1.8, A260/A230 > 1.8). The obtained miRNA was stored at $-80^{\circ} \mathrm{C}$ until use.

Single-stranded complementary DNA (cDNA) was obtained from $1 \mu \mathrm{g}$ of purified miRNA using the SensiFAST ${ }^{\mathrm{TM}}$ cDNA Synthesis Kit (Bioline, Catalog number BIO-65053, London, UK).

All PCR runs were performed on the Applied Biosystems Step One ${ }^{\mathrm{TM}}$ Instrument. The samples of the same group were run together to avoid any interrun variety. The preliminary tests were performed to determine the optimal condition for the used primer sequences (Table 1). The miRNA expression level was then calculated as described by Yuan et al. (2016) using Applied Biosystems Step One Instrument software. The obtained qRTPCR data were analyzed by plotting the expression of the dedicated genes using the expression of $2^{-\Delta \Delta C T}$ as extracted from amplification curves by the Rotor-Gene Q Series Software 2.0.3 (Qiagen, Hilden, Germany).

\section{Dielectric relaxation studies}

Silver electrode cells (1 ml volume) were used for the dielectric measurements for the blood sample for normal person, $\mathrm{HCV}$ patient, and $\mathrm{HCV}$-treated patients with different doses of 
Sovaldi. The capacitance C, impedance Z, and dielectric loss tangent tan_and dielectric loss tangent tan oses of ic mea and 5 MHz using HIOKI 3532 LCR, a programmable automatic RCL meter.

\section{Models and data analysis of dielectric relaxation}

A dielectric spectroscopy has the sensitivity for dynamical operations that include the reorientation of dipolar structures or displacement of charged elements, which can lead to dispersive behavior or even loss of the dielectric constant.

The real $\left(\varepsilon^{\prime}\right)$ and imaginary $\left(\varepsilon^{\prime \prime}\right)$ parts of dielectric constant and dielectric loss $(\tan \delta)$ are calculated using the following relations:

$$
\varepsilon^{\prime}=C_{\mathrm{p}} d / \varepsilon_{0} A
$$

where $C_{\mathrm{p}}$ is capacitance in farad $(F), A$ is cross-sectional area of pallet in $\mathrm{m}^{2}, d$ is the thickness of the pallet, and $\varepsilon_{0}$ is the permittivity in free space.

$\varepsilon^{\prime \prime}=\varepsilon^{\prime} \tan \delta$

$\left(\sigma_{\mathrm{ac}}\right)$ is calculated using the standard realtion

$$
\text { бac }=2 \pi f \varepsilon_{0} \varepsilon^{\prime} \tan \delta
$$

where $f$ is frequency of applied field in $\mathrm{Hz}$.

A complex impedance plot can be represented by dielectric loss $\tan \delta$

$$
\tan \delta=\mathrm{z}^{\prime} / \mathrm{z}^{\prime \prime}=\varepsilon^{\prime} / \varepsilon^{\prime \prime}
$$

The dielectric constant and loss factor can be calculated

by

$$
\varepsilon^{\prime}=\varepsilon_{\infty}+\frac{\varepsilon_{S}-\varepsilon_{\infty}}{1+\omega^{2} \tau^{2}}
$$

Table 1. Primer sequences used in the study.

\begin{tabular}{ll}
\hline Gene & Rat primer sequence (5'-3') \\
\hline \multirow{2}{*}{-actin gene } & Forward 5'-GCA CCA CAC CTT CTA CAA TG-3' \\
& Reverse 5'-TGC TTG CTG ATC CAC ATC TG-3' \\
MiR-21 & Forward 5'-GCCCGCTAGCTTATCAGACTGATG-3' \\
& Reverse 5'-GTGCAGGGTCCGAGGT-3' \\
MiR-155 & Forward 5-TGTATGCTGTTAATGCTAATTGTGA-3 \\
& Reverse 5-TGTTAATGCTAACAGGTAGGAGTC-3 \\
MiR-122 & Forward 5-TCTGGAGTGTGACAATGGTGT-3 \\
& Reverse 5-CCTAGCAGTAGCTGTTAGTGTG-3 \\
MiR-205 & Forward 5-ATCCTCAGACAATCCATGTGCT-3 \\
& Reverse 5-ACTCCACTGAAATCTGGTTGGG-3 \\
MiR-133a & Forward 5-GGAGCCAAATGCTTTGCTAGA-3 \\
& Reverse 5-CGCCATCAATGCACAGCTAC-3
\end{tabular}

All primers sequences were obtained from the Gene Bank of NCBI BLAST table.

$$
\varepsilon^{\prime \prime}=\left(\varepsilon_{S}-\varepsilon_{\infty}\right) \frac{\omega \tau}{1+\omega^{2} \tau^{2}}
$$

where $\varepsilon^{\prime}$ is the real part of the dielectric permittivity that represents the stored energy, whereas $\varepsilon^{\prime \prime}$ is the imaginary part of dielectric permittivity associated with the dissipation energy (or loss of energy) within the medium, $\varepsilon \infty$ is the dielectric constant or the permittivity value at the end of the dispersion, at frequencies greater than $1 /(2 \pi \tau)$, $\varepsilon s$ is the static or very low frequency or dielectric constant, $\tau$ is the relaxation time $(=1 / 2 \pi \mathrm{fc})$, and $\mathrm{fc}$ is the characteristic frequency (Pethig, 1979).

\section{Statistical analysis}

The statistical analysis of data was performed using GraphPad InStat, version 6.01 (GraphPad Software Inc., San Diego, CA). The obtained data were expressed as mean \pm standard deviation (SD). The significant differences between the means were determined by the one-way analysis of variance test. $p$-value less than 0.05 was considered to be statistically significant. The graphs were plotted using Microsoft Excel 2010.

\section{RESULTS}

The results revealed that miR-21 and miR-155 concentrations were significantly increased in HCV patients who did not receive any treatment $(\mathrm{G} 2)$ compared to the negative control (G1). The relative concentrations of miR-21 and miR155 gradually decreased with Sovaldi treatment to reach to nearly normal values. The concentrations of miR-205 showed also upregulation by the same manner of miR-21 and miR-155. However, after treatment with three doses of Sovaldi, miR-205 concentrations showed a good regression with slightly elevation than normal values. On the other hand, miR-122 and miR-133a concentrations were downregulated in $\mathrm{HCV}$ patients who did not receive any treatment (G2) if compared to G1. After treatment with Sovaldi (G3 or G4), the data obtained showed an elevation in miR-122 concentrations in comparison with G2, whereas, in G5 (patients who received 3 doses of Sovaldi), significant elevation in miR-122 expression compared to G2 was achieved. The miR-122 and miR-133a relative concentrations of G5 were very close to normal values (Table 2).

The impedance data investigation is regularly done by a complex plane way that basically includes plotting the impedance imaginary part against the real part $\left(-Z_{i m g} v s . Z_{\text {real }}\right)$. Thus, different equivalent circuits can be determined from the plots in Figure $1 \mathrm{a}$. The electrical properties of materials can be described by relative permittivity $\left(\varepsilon_{\mathrm{r}}\right)$. The relative dielectric blood constant is calculated from the ratio of the blood permittivity $(\varepsilon)$ and vacuum dielectric

Table 2. Assessment of different miRNA types relative to $\beta$-actin housekeeping gene in serum of all groups.

\begin{tabular}{cccccc}
\hline Group & miR-21 & miR-155 & miR-205 & miR-122 & miR-133a \\
\hline G1 volunteers & $1.00 \pm 0.0$ & $1.00 \pm 0.0$ & $1.00 \pm 0.0$ & $1.00 \pm 0.0$ & $1.00 \pm 0.0$ \\
G2 (zero time) & $5.74 \pm 1.92^{\mathrm{a}}$ & $4.33 \pm 1.07^{\mathrm{a}}$ & $6.05 \pm 2.18^{\mathrm{a}}$ & $0.37 \pm 0.17 \mathrm{a}$ & $0.46 \pm 2.46^{\mathrm{a}}$ \\
G3 (1st dose) & $3.86 \pm 2.31^{\mathrm{a}, \mathrm{b}}$ & $3.24 \pm 1.90^{\mathrm{a}, \mathrm{b}}$ & $4.22 \pm 1.89^{\mathrm{a}, \mathrm{b}}$ & $0.48 \pm 0.12^{\mathrm{a}}$ & $0.53 \pm 0.15^{\mathrm{a}}$ \\
G4 (2nd dose) & $2.75 \pm 1.47^{\mathrm{a}, \mathrm{b}}$ & $2.16 \pm 0.89^{\mathrm{a}, \mathrm{b}}$ & $3.52 \pm 1.47^{\mathrm{a}, \mathrm{b}}$ & $0.59 \pm 0.06^{\mathrm{a}, \mathrm{b}}$ & $0.72 \pm 0.08^{\mathrm{a}, \mathrm{b}}$ \\
G5 (3rd dose) & $1.80 \pm 1.64^{\mathrm{b}}$ & $1.22 \pm 0.73^{\mathrm{b}}$ & $2.08 \pm 1.39^{\mathrm{a}, \mathrm{b}}$ & $0.83 \pm 0.17^{\mathrm{b}}$ & $0.80 \mathrm{c} \pm 0.16^{\mathrm{b}}$ \\
\hline
\end{tabular}

Data are expressed as mean $\pm \mathrm{SD}$. The symbols a and $\mathrm{b}$ indicate a significant change $(p<0.05)$ in comparison with G1 (healthy volunteers, negative control) and G2 (HCV patients, positive control), respectively. 


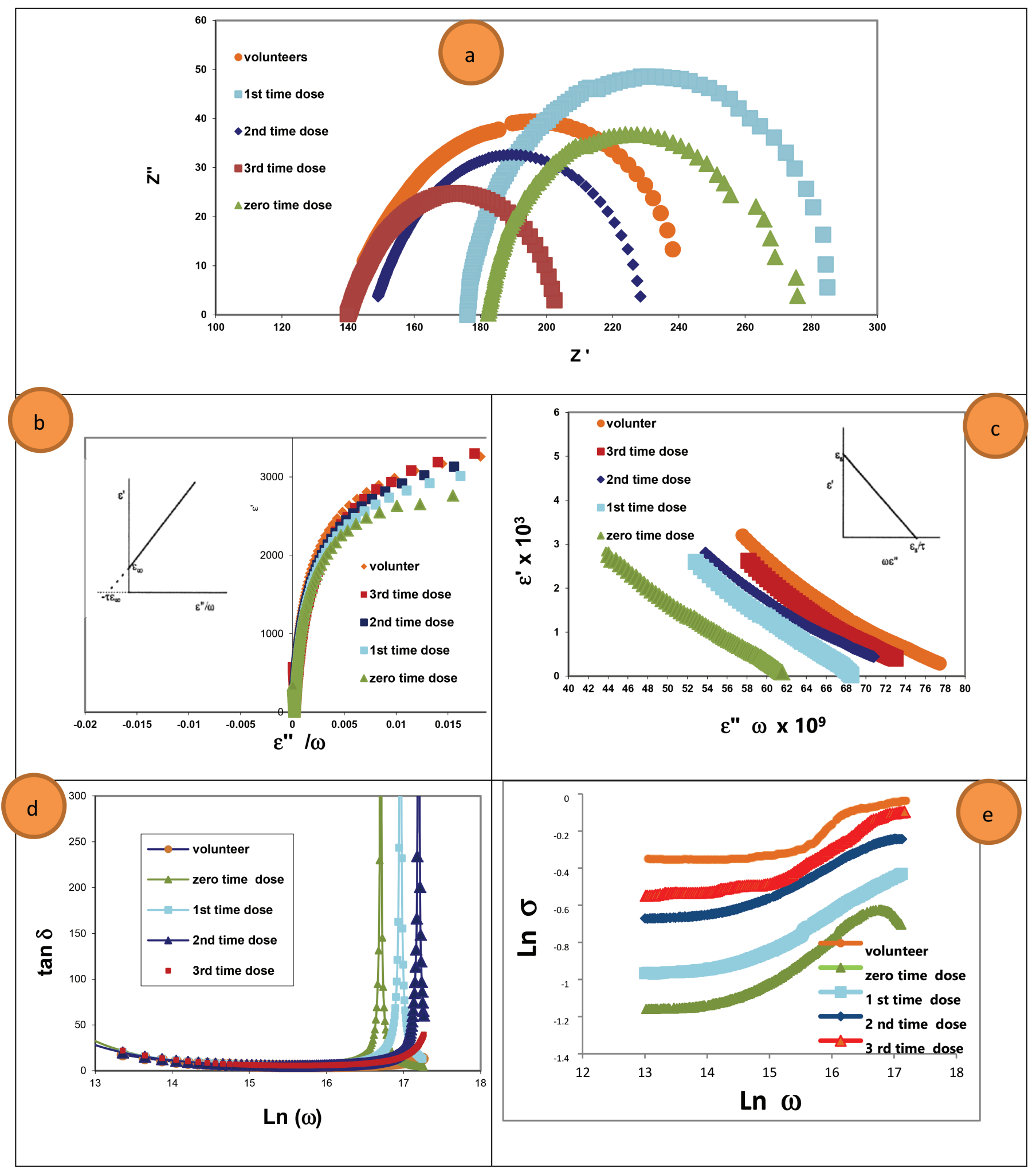

Figure 1. (a) Cole-Cole plot (Variation of $z^{\prime}$ with $z^{\prime \prime}$ ), (b) Variation of $\varepsilon^{\prime}$ with $\varepsilon^{\prime \prime} \omega$, (c) Variation of $\varepsilon^{\prime}$ with $\varepsilon^{\prime \prime} / \omega$, (d) Dielectric loss (tan $\delta$ ) as a function of frequency and (e) Variation of $\log \sigma a c$ with $\log \omega$ for normal person, HCV-patient and $\mathrm{HCV}+$ sovaldi with different doses.

constant $\left(\varepsilon_{0}\right)$. The blood conductivity increased gradually and slowly in lower frequency range, whereas the relative dielectric constant decreases rapidly. In the cases of the frequency higher than $10^{5} \mathrm{~Hz}$, the relative dielectric constant reduced rapidly compared to cases, in which the frequency was lower than $10^{6} \mathrm{~Hz}$. However, its conductivity was elevated by a faster way when the 
Table 3. The mean variations of fitting parameters $\left(R_{o,} R_{\infty}, \varepsilon_{s}, \varepsilon_{\infty}\right.$, and $\left.\tau\right)$ for all group blood samples.

\begin{tabular}{cccccc}
\hline Group & $\boldsymbol{R}_{\infty}$ & $\boldsymbol{R}_{\boldsymbol{\theta}}$ & $\boldsymbol{\varepsilon}_{s}$ & $\boldsymbol{\varepsilon}_{\infty}$ \\
\hline G1 (volunteers) & $140.0 \pm 3.54$ & $240.0 \pm 6.43$ & $5,800 \pm 20.43$ & $1,800 \pm 19.44$ \\
G2 (zero time) & $181.33 \pm 5.53$ & $280.0 \pm 7.63$ & $3,250 \pm 21.49$ & $1,500 \pm 14.95$ & $6.67 \mathrm{E}-06$ \\
G3 (1st dose) & $175.83 \pm 4.24$ & $285.8 \pm 7.73$ & $4,500 \pm 18.53$ & $1,600 \pm 15.73$ & $6.55 \mathrm{E}-06$ \\
G4 (2nd dose) & $148.33 \pm 2.64$ & $230.17 \pm 5.33$ & $5,000 \pm 19.47$ & $1,650 \pm 16.42$ & $6.67 \mathrm{E}-06$ \\
G5 (3rd dose) & $139.33 \pm 2.73$ & $202.17 \pm 4.23$ & $5,480 \pm 16.63$ & $1,700 \pm 17.33$ & $6.76 \mathrm{E}-06$ \\
\hline
\end{tabular}

frequency was more than $10^{5} \mathrm{~Hz}$ (Fig. 1). From the variation of _" with_wi and with ith for normal person, $\mathrm{HCV}$ patient, and $\mathrm{HCV}$ + Sovaldi with different doses, we can calculate $\varepsilon_{\infty}$ which is the dielectric constant at frequencies more than $1 \ldots, \varepsilon_{\mathrm{s}}$ is the static or very low frequency or dielectric constant, and $\tau$ is the relaxation time. The huge increase in the high frequency of dielectric loss $\left(\tan \delta\right.$ ) when the frequency is more than $10^{5} \mathrm{~Hz}$ in $\mathrm{HCV}$ patients and HCV + Sovaldi with different doses indicated that the blood molecules underwent a very significant conformational change. The values of $\mathrm{R}_{0}$ and $\mathrm{R}_{\infty}$ for $\mathrm{HCV}$ patients are higher than those for healthy volunteers. Then, it gradually decreased after first and second doses of treatment till reaching to nearly normal values after the third dose, whereas relaxation time _ increased by Sovaldi treatment (Table 3).

\section{DISCUSSION}

The crosstalk between miRNA and HCV is a rising area of investigation. Circulating miRNAs could be developing study zone, but there are many challenges appearing in understanding the cellular bases and circulating miRNAs roles in HCV infection and related liver diseases. Recent studies suggested that miRNAs can be used as predictive, diagnostic, or prognostic biomarkers during $\mathrm{HCV}$ infection. Several proofs indicate that miRNAs have effects on host immunity to $\mathrm{HCV}$ infection and clinical consequences of standard HCV treatment. On the other hand, HCV has developed different plans to interrupt the interferon signaling pathway to facilitate its own replication and regulate $\mathrm{HCV}$ genomic RNA abundance (Hou et al., 2010). This evade occurs by stimulating miRNAs that control the production of target genes involved in innate immune response improvement against viral infections (Shrivastava and Ray, 2014). The obtained results in this study showed a dramatic decrease in patients who did not receive any treatment $(\mathrm{G} 2)$ in miR-122 gene expression levels to reach to about one-third of its normal levels. These levels increased gradually by the followed treatment strategy to reach to its nearly normal values in patients who received treatment for 3 successive months (G5). The previous studies indicated a reverse relationship between hepatic miR-122 and HCV-RNA within the liver and serum (Choi et al., 2013). MiR122 represents a great successful target for improving hepatic steatosis besides many studies that documented miR122 as a helpful target in HCV-targeted therapy through its function in neutralizing antagomir generating (Elemeery, 2018). MiR-133a results showed the same manner. The gene expression of miRNA-133a in patients who did not receive any treatment $(\mathrm{G} 2)$ also significantly decreased to about less than one-half of its normal values. This decrease was gradually regressed by following the treatment course to reach to about 0.8 of its normal values in patients who completed the treatment course (G5). This can be discussed by the growing evidence which has illustrated that miR-133a is an imperative controller of hepatocellular carcinoma (HCC) (Liang et al., 2018). The main target genes for miR-133a are human collagen alpha-3 (V) chain (COL5A3), epidermal growth factor receptor (EGFR), insulin like growth factor-1 receptor (IGF1R), chicken tumor virus regulator of kinase (CRK), and collagen type VI, $\alpha 3$ (COL6A3) which are specifically ECM-receptor interaction. Both miR-122 and miR133a are classified as downregulated miRNA families. They were suggested to have roles in hepatic stellate cells activation and fibrogenesis. The ECM-receptor interaction class predominantly comprises ECM components, integrins, and laminins. Moreover, $\mathrm{HCV}$ infection is related to an miRNA expression model in liver that inhibits cellular adhesion and ECM genes, which participate in HCV-mediated fibrogenesis (Bandyopadhyay et al., 2011).

On the other hand, miR-155, miR-205, and miR-21 gene expression results showed an extremely significant upregulation in all HCV patients who did not receive any treatment (G2). Many previous studies indicated the elevation in miR-155 expression in Kupffer cells after any inflammation, especially HCV. This occurred because the main target gene of miR-155 is tumor necrosis factors which can also promote HCC through suppressed adenomatous polyposis coli and subsequently stimulated Wnt signaling, which in turn causes hepatocyte proliferation and tumorogenesis (Roy et al., 2015; Zhang et al., 2012), whereas miR-21 results can be explained by the HCV upregulation of miR-21 which in turn inhibits interferon (IFN) signaling inside cell during $\mathrm{HCV}$ infection, thus progressing the infection. In normal conditions, the activated hepatic cells produce proinflammatory cytokines and interferons against viral infection. This can occur at the recognition of viral components by pattern recognition receptors, such as the toll-like receptors (TLRs) and retinoic acid-inducible gene I-like helicases. These pathways are strongly controlled by the host to avoid an improper cellular response, but viruses can modify these pathways to replicate. A previous study indicated HCV strategy, where it escapes from the immune system control to proliferate by stimulating miR-21 via several viral proteins and signaling components. Furthermore, miR-21 targets two main factors in the TLR signaling pathway, such as myeloid differentiation factor 88 (MyD88), and interleukin-1 receptor-associated kinase 1 (IRAK1), which are included in HCV-induced type I IFN expression. Furthermore, a transcription factor, activating protein-1, causes miR-21 stimulation due to $\mathrm{HCV}$ infection via $\mathrm{PKC} / \mathrm{JNK} / \mathrm{c}-J u n$ and $\mathrm{PKC} \alpha / \mathrm{ERK} / \mathrm{c}-\mathrm{Fos}$ cascades. Briefly, miR-21 expression is stimulated during $\mathrm{HCV}$ infection, and it downregulates IFN- $\alpha$ signaling via MyD88 and IRAK1 (Chen et al., 2013). On contrary to the results, a previous study found that hepatitis-B downregulated miR-205 by activating hypermethylation of miR-205 promoter (Zhang et al, 2013). In general, miR-205 has many dual complex roles in different processes as inflammation, tumor 
initiation, and progression. For example, MiR-205 may act either as a tumor suppressor by preventing reproduction and invasion or as an oncogene by stimulating tumor initiation and proliferation, according to the specific tumor conditions and target genes and the same mechanism for other processes as inflammation (Qin et al., 2013). The upregulation of miR-205 suppresses suppressor of mothers against decapentaplegic homolog 4 (SMAD4) and Phosphatase and tensin homolog (PTEN) (the key mediators of TGF- $\beta$ pathway), which were identified as potential target genes for miR-205 (Li et al., 2017).

The conductivity and dielectric constant of all blood samples result from the relaxation of the dipoles with a Debye type. The relaxation time changed in all samples since the blood red cells (or any large molecule) are polarized at external AC electric current application. Thus, this AC electric current was transferred via the blood. During the polarization steps, the normal blood molecules relaxed with a relaxation time $\tau_{n}$, and the HCV ones relaxed with a relaxation time $\tau_{H}$. These times are related to the internal energy of these molecules (Knocks and Weingärtner, 2001). As the larger the structure, the longer the relaxation time, many relaxation mechanisms can occur. Any particle with its natural relaxation time $\tau$ will have a probability $\omega \tau$ to respond to the applied field; if $\tau>1 / \omega$, where $\omega=2 \pi \mathrm{f}$ and $\mathrm{f}$ is the applied frequency, low response will be achieved; similarly, if $\tau<1 / \omega$, low response also will be achieved. However, when $\tau=1 / \omega$; maximal strongest response will be achieved (Doster et al., 2010). The peaks of dielectric loss tangent for normal person, HCV patient, and $\mathrm{HCV}$-treated patients with different doses are shifted to higher frequency with time and disappeared in healthy volunteers and in patients who completed the treatment course. Free radical increment in the HCV patient blood enhances the dipoles density, which in turn increases the dielectric constant, whereas HCV treated with Sovaldi showed a decrease in blood cell dielectric constant after third dose and reached to nearly normal values. In physical expressions, the electrical conductivity of blood can be determined by the measurement of its internal charge ability to transfer the electrical energy from one electrode to another. Similarly, the blood permittivity is a measurement of its charged dipoles storage ability of the applied external energy (Feldman et al., 2003; Oleinikova et al., 2004).

\section{CONCLUSION}

This study evaluated changes occurring in different types of miRNA gene expression and some blood electrical parameters after HCV infection and during Sovaldi administration till the end of treatment course ( 3 months). The results showed that the studied types of miRNAs (MiR-21, Mir-155, MiR-205, Mir-133a, and MiR-122) and the different bioimpedance measurements as electrical conductivity and blood permeability represent good and promising tools for the noninvasive studying of blood internal structure. They can play key roles in monitoring physiological changes and help in the early diagnosis and prognosis of the development and progression of $\mathrm{HCV}$.

\section{CONFLICT OF INTEREST}

Authors declared that they do not have any conflicts of interest.

\section{FINANCIAL SUPPORT AND SPONSORSHIP}

There is no financial support received for this research.

\section{REFERENCES}

Ahmed OA, Safwat E, Khalifa MO, Elshafie AI, Fouad MHA, Salama MM, Naguib GG, Eltabbakh MM, Sherief AF, Abd-Elsalam S. Sofosbuvir plus daclatasvir in treatment of chronic hepatitis $\mathrm{C}$ genotype 4 infection in a cohort of Egyptian patients: an experiment the size of Egyptian village. Int J Hepatol, 2018; 1:1-5; doi:10.1155/2018/9616234

Bandyopadhyay S, Friedman RC, Marquez RT, Keck K, Kong B, Icardi MS, Brown KE, Burge CB, Schmidt WN, Wang Y, McCaffrey AP. Hepatitis $C$ Virus infection and hepatic stellate cell activation Downregulate miR-29: miR-29 overexpression reduces Hepatitis C Viral abundance in culture. J Infect Dis, 2011; 203(12):1753-62.

Barshtein G, Manny N, Yedgar S. Circulatory risk in the transfusion of red blood cells with impaired flow properties induced by storage. Transfus Med Rev, 2011; 25:24-35.

Bartel DP. MicroRNAs: target recognition and regulatory functions. Cell, 2009; 136:215-33.

Ben-Ishai P, Talary MS, Caduff A, Levy E, Feldman Y. Electrode polarization in dielectric measurements: a review. Meas Sci Technol, 2013; 24:1-21; doi:10.1088/0957-0233/24/10/102001

Chen Y, Chen J, Wang H, Shi J, Wu K, Liu S, Liu Y, Wu J. HCVInduced miR-21 contributes to evasion of host immune system by targeting MyD88 and IRAK1. PLoS Pathog, 2013; 9(4):e1003248.

Choi Y, Dienes HP, Krawczynski K. Kinetics of miR-122 expression in the liver during acute HCV infection. PLoS One, 2013; 8(10):e76501.

Doster W, Busch S, Gaspar AM, Appavou, MS, Wuttke J, Scheer H. Dynamical transition of protein-hydration water. Phys Rev Lett, 2010; 104(9):098101.

El-Ahwany EG, Mourad L, Zoheiry MK, Abu-Taleb H, Hassan M, Atta R, Hassanien M, Zada S. MicroRNA-122a as a non-invasive biomarker for $\mathrm{HCV}$ genotype 4-related hepatocellular carcinoma in Egyptian patients. Arch Med Sci, 2019; 15(6):1454-61.

Elemeery MN. MicroRNA in hepatocellular carcinoma - related Hepatitis C Virus patients in correlation to disease progression, Hepatitis C - from infection to cure. IntechOpen, London, UK; chapter 5, pp 87-100, 2018.

El-Ghitany EM. Hepatitis C Virus infection in Egypt: current situation and future perspective. J High Inst Public Health, 2019; 49(1):1-9.

Feldman Y, Ermolina I, Hayashi Y. Time domain dielectric spectroscopy study of biological systems, IEEE Trans Dielectr Electr Insul, 2003; 10(5):728-53.

Hou W, Tian Q, Zheng J, Bonkovsky HL. MicroRNA-196 represses Bach1 protein and hepatitis $\mathrm{C}$ virus gene expression in human hepatoma cells expressing hepatitis C viral proteins. Hepatology, 2010; 51:1494-504.

Jaferzadeh K, Moon I. Quantitative investigation of red blood cell three-dimensional geometric and chemical changes in the storage lesion using digital holographic microscopy. J Biomed Opt, 2015; 20(11): 111218

Knocks A, Weingärtner $\mathrm{H}$. The dielectric spectrum of ubiquitin in aqueous solution. J Phys Chem B, 2001; 105(17):3635-8.

Lee $\mathrm{CH}$, Kim JH, Lee SW. The role of MicroRNA in pathogenesis and as markers of HCV chronic infection. Curr Drug Targets, 2017; 18(7):756-65.

Li J, Hu K, Gong G, Zhu D, Wang Y, Liu H, Wu X. Upregulation of MiR-205 transcriptionally suppresses SMAD4 and PTEN and contributes to human ovarian cancer progression. Sci Rep, 2017; 7:41330-9.

Liang H, Yang X, Wen D, Gao L, Zhang X, Ye Z, Luo J, Li Z, He Y, Pang Y, Chen G. Utility of miR-133a-3p as a diagnostic indicator for hepatocellular carcinoma: an investigation combined with GEO, TCGA, meta-analysis and bioinformatics. Mol Med Rep, 2018; 17(1):1469-84. 
Livshits L, Caduff A, Talary MS, Feldman Y. Dielectric response of biconcave erythrocyte membranes to D- and L-Glucose. J Phys D Appl Phys, 2006; 40(1):15.

Oleinikova A, Sasisanker P, Weingartner $\mathrm{H}$. What can really be learned from dielectric spectroscopy of protein solutions? A case study of ribonuclease A. J Phys Chem B, 2004; 108:8467-74.

Pethig R. Dielectric and electronic properties of biological materials. John Wiley \& Sons, New York, NY, p376, 1979.

Qin AY, Zhang XW, Liu L, Yu JP, Li H, Wang SZ, Ren XB, Cao S. MiR-205 in cancer: an angel or a devil? Eur J Cell Biol, 2013; 92(2):54 60.

Roy S, Benz F, Luedde T, Roderburg C. The role of miRNAs in the regulation of inflammatory processes during hepatofibrogenesis. Hepatobilary Surg Nutr, 2015; 4(1):24-33.

Shrivastava S, Ray RB. Hepatitis C virus infection, autophagy and innate immune response. In: Hayat MA (ed.), Autophagy. Cancer, other pathologies, inflammation, immunity, infection and aging, vol. 3, Academic Press, Elsevier, Cambridge MA, pp 164-90, 2014.

Shrivastava S, Steele R, Ray R, Ray RB. MicroRNAs: role in hepatitis $C$ virus pathogenesis. Genes Dis, 2105; 2(1):35-45.
Yuan L, Ke Z, Ma J, Guo Y, Li Y. IRGM gene polymorphisms and haplotypes associate with susceptibility of pulmonary tuberculosis in Chinese Hubei Han population. Tuberculosis (Edinb), 2016; 96:58-64.

Zhang T, Zhang J, Cui M, Liu F, You X, Du Y, Gao Y, Zhang S, Lu Z, Ye L, Zhang X. Hepatitis B virus X protein inhibits tumor suppressor miR-205 through inducing hypermethylation of miR-205 promoter to enhance carcinogenesis. Neoplasia, 2013; 15:1282-91.

Zhang Y, Wei W, Cheng N, Wang K, Li B, Jiang X, Sun S. Hepatitis C virus-induced up-regulation of microRNA-155 promotes hepatocarcinogenesis by activating Wnt signaling. Hepatology, 2012; 56:1631-40.

How to cite this article:

Beltagy DM, Khedr YI, Sakr AHA. Diagnostic and prognostic roles of miRNA and dielectric parameters in HCV Egyptian patients. J Appl Pharm Sci, 2020; 10(09):115-121. 